\title{
DETERMINACIÓN Y ESTANDARIZACIÓN DE LAS MEJORES CONDICIONES DE PROCESO DE LAVADO CIP (CLEANING IN PLACE): EN LA ZONA DE EVAPORACIÓN DE LA PLANTA PULVERIZADORA DE LECHE COLANTA PLANETA RICA
}

\section{DETERMINATION AND STANDARDIZATION OF THE TOP CONDITIONS OF WASH CIP (CLEANING IN PLACE) PROCESS: EVAPORATION ZONE IN THE SPRAY MILK PLANT COLANTA PLANETA RICA}

\author{
Omar A. Pérez ${ }^{1}$, Ramiro Torres ${ }^{2}$, Everaldo J. Montes ${ }^{3}$, Ricardo D. Andrade ${ }^{4}$, Vivian P. Argel ${ }^{5}$, Alcides S. \\ Durango ${ }^{6}$, Edwin Negrete ${ }^{7}$.
}

Recibido para publicación: 21 de junio de 2012 - Aceptado para publicación: 17 de Noviembre de 2012

\section{RESUMEN}

La industria lechera utiliza desde hace muchos años el CIP (cleaning in place) como método de limpieza. En la planta Pulverizadora Colanta Planeta Rica no existía un procedimiento de CIP que garantizara la eficiencia y condiciones constantes durante el proceso. Mediante modificaciones de infraestructura en los equipos, recomendaciones de los proveedores, revisión bibliográfica y ensayos realizados en la planta se logró determinar y estandarizar las condiciones para desarrollar el proceso CIP como; concentraciones y temperaturas de las soluciones de lavado, caudales y tiempos de proceso. Se logró que las soluciones de lavado recircularan a las concentraciones deseadas (soda caustica de $3-4 \%$ y ácido nítrico de $2-$ $3 \%)$. Se consiguió mantener la temperatura de la soda en un rango de 70 a $80^{\circ} \mathrm{C}$ durante su recirculación en los efectos del evaporador. Se alcanzó el caudal máximo posible para realizar el CIP en el pasterizador el cual garantizara el arrastre total de partículas adheridas y se determinó el caudal más adecuado para el lavado del evaporador que garantizara una

1 Ph.D Ciencia y tecnología de alimentos, Profesor Asociado Universidad de Córdoba, Montería - Colombia, oaperez@correo.unicordoba.edu.co

2 Ms.C Ciencias Agroalimentarias, Profesor Asistente Universidad de Córdoba, Montería - Colombia, rtorres@correo.unicordoba.edu.co

3 Ms.C Ciencia y tecnología de alimentos, Profesor Titular Universidad de Córdoba, Montería - Colombia, everraldomontes@yahoo.com

${ }_{5}^{4} \mathrm{Ph} . \mathrm{D}$ (c) en Ciencia y tecnología de alimentos, Profesor Asociado Universidad de Cordoba, Monteria - Colombia

${ }_{6}^{5}$ Estudiante Ingeniería de Alimentos, Universidad de Córdoba, Montería - Colombia

Ingeniero de Alimentos, Coordinador de Producción de la Planta Colanta Planeta Rica, alcidesdc@colanta.com.co

${ }^{7}$ Ingeniero de Alimentos, Coordinador de Producción de la Planta Colanta Planeta Rica 
limpieza eficiente y evitara la inundación del equipo. Se logró la reducción del CIP de 6 a 4,5 horas representando una ganancia de $\$ 748^{\prime} 459.008$ anuales, ya que el tiempo ahorrado es ahora invertido en la producción de leche en polvo, lo cual estuvo mayoritariamente influenciado por la reducción del tiempo de recirculación de soda en el pasterizador, el cual no se había bajado de 3 horas pese a la sospecha que pudiera influir negativamente en el equipo o en el proceso productivo.

Palabras clave: CIP, evaporador, pasterizador, soda caustica.

\section{ABSTRACT}

The dairy industry has used for many years the CIP as method of cleanliness. In the Spray Milk Plant Colanta Planeta Rica did not exist CIP procedure which guarantee the efficiency and constant conditions during the process. Through modifications of infrastructure in the equipments, recommendations of the suppliers, bibliographical review and tests realized in the plant, it was determine and standardized the conditions to develop the process CIP such as; concentrations and temperatures of wash solution, flows and process times. It was achieved that wash solutions re-circulate the desired concentrations (caustic soda of $3-4 \%$ and nitric acid of $2-3 \%$ ), the temperature of the soda was kept in a range from 70 to $80{ }^{\circ} \mathrm{C}$ during its recirculation in the effects of the evaporator, it was reached the maximum flow possible to realize the CIP in the pasteurizer which guarantee the total dragging of adhered particles and it was determinate the most suitable flow for the wash of the evaporator which guarantee an efficient cleanliness and avoid the flood of the equipment. The reduction of the CIP was achieved from 6 to 4,5 hours representing a profit of $\$ 748$ ' 459.008 annual, since the saved time is now inverted in the production of powdered milk, this was influenced mostly by the reduction of the recirculation time of soda in the pasteurizer, which had not being dropped from 3 hours in spite of the suspicion that it could influence negatively in the equipment or the productive process.

Keywords: CIP, evaporator, pasteurizer, caustic soda

\section{INTRODUCCIÓN}

De acuerdo a las exigencias legales vigentes en el país sobre los requisitos que debe cumplir la leche para consumo humano, referenciadas en el decreto 616 del 28 de febrero del 2006, se manifiesta que debe adoptarse con carácter obligatorio el sistema de análisis de peligros y control de puntos críticos HACCP, cuyos procedimientos para la implementación y certificación, están basados en el decreto 60 del 18 de enero del 2002 y las normas que lo modifiquen. Para la implementación del sistema HACCP es necesario adoptar un adecuado plan de limpieza y desinfección que garantice la higiene de los equipos con el fin de no poner en riesgo el producto elaborado. La industria láctea utiliza hace varios años el sistema CIP (Cleaning in Place) como método de limpieza; en el que se consigue mezclar en proporciones adecuadas el agua y los productos químicos a la temperatura ideal, para asegurar una máxima eficiencia y permite sanitizar todas las instalaciones sin necesidad de desmontarlas y con el mínimo requerimiento manual [6]

EI CIP implementado para la limpieza de la planta pulverizadora Colanta Planeta Rica se ha realizado durante muchos años bajo condiciones no estandarizadas, lo que genera una constante variación de las actividades 
efectuadas, un inadecuado control de los tiempos de lavado, de las concentraciones de las soluciones empleadas, de las condiciones de uso del detergente, de las temperaturas y caudales del proceso; traduciéndose en un incremento de tiempo perdido por extensión del lavado, no aprovechable para la producción de leche en polvo que genera a largo plazo una pérdida económica. Los dos equipos principales de la zona de pulverización de la planta Colanta Planeta Rica, son un pasterizador en serpentin y un evaporador a vacio de película descendente de 4 efectos, los cuales anteriormente conformaban un solo circuito para realizar el CIP, con algunas modificaciones de infraestructura, se logró independizar el evaporador del pasterizador con el fin de realizar el CIP de manera simultánea e independiente en los dos equipos.

Dentro de las variables objeto de este estudio se encuentran, las concentraciones y temperaturas de las soluciones de lavado, los caudales y tiempos de proceso.

La concentración de las soluciones de lavado (soda caustica y ácido nítrico) durante la recirculación en los equipos deben ajustarse correctamente antes de que empiece la limpieza, ya que durante la misma las soluciones se diluyen con el agua de enjuagado o con los restos de leche. Se recomienda que los detergentes ácidos recirculen a una concentración entre 2 y $3 \%$, mientras que los detergentes alcalinos entre 3 y $4 \%$ ya que concentraciones inferiores disminuyen la eficiencia del proceso [2].

Al igual que las concentraciones de las soluciones de lavado, las temperaturas empleadas durante la recirculación de las mismas tienen gran influencia en la calidad del proceso. La efectividad de la solución detergente, se incrementa conforme aumenta la temperatura, aunque la solución siempre tiene una temperatura óptima a la cual debe ser utilizada, elevándose la temperatura se consiguen los siguientes efectos: disminución de la fuerza de atracción entre las impurezas y las superficies, disminución de la viscosidad e incremento de la acción turbulenta, incremento de la solubilidad de los materiales solubles e incremento de la velocidad de reacción química. Como regla general la limpieza con detergentes alcalinos, se debe realizar a la misma temperatura que el producto haya sido expuesto, siendo el mínimo unos $70^{\circ} \mathrm{C}$. Se recomiendan temperaturas de 68 a $70^{\circ} \mathrm{C}$ con detergentes ácidos y de 70 a $80^{\circ} \mathrm{C}$ con detergentes alcalinos (Sc 2004).

El efecto mecánico en los procesos de CIP se consigue mediante una adecuada velocidad de flujo de las soluciones de limpieza. Las bombas de alimentación del detergente se dimensionan para conseguir mayores capacidades que para el transporte del producto, con velocidades de flujo de 1.5 - 3 $\mathrm{m} / \mathrm{s}$ en las tuberías. A estas velocidades el líquido fluye de forma muy turbulenta lo cual da lugar a un buen efecto de limpieza sobre las superficies de los equipos [7]

El tiempo necesario para la limpieza depende del espesor de los depósitos de suciedad y de la temperatura de la solución de detergente. La limpieza puede ser incrementada con el uso de tiempos largos, sin embargo un aumento del tiempo más allá de un valor dado proporciona un pequeño y adicional incremento en la efectividad [8]

El tiempo del proceso de lavado CIP está mayoritariamente limitado por la recirculación de soda en el pasterizador. La temperatura de pasterización del proceso alcanza los $92^{\circ} \mathrm{C}$ lo cual facilita la formación de una capa gruesa de costra de leche en el serpentín del pasterizador a medida que avanza la corrida de proceso, la cual hay que remover totalmente durante el aseo para poder iniciar sin dificultad una nueva corrida de leche en polvo[6].

En el evaporador no hay lugar a este tipo de problemas ya que el equipo opera al vacio y las temperaturas no sobrepasan los $70^{\circ} \mathrm{C}$ y por ende no hay formación de costra leche, por tal motivo el tiempo de recirculación de soda en el pasterizador debe ser superior al tiempo de recirculación de soda en el evaporador para que el equipo quede totalmente limpio.

Con el fin de disminuir el tiempo total del proceso de CIP, se determinó el menor tiempo de recirculación de soda en el pasterizador el cual garantizara una limpieza eficiente y así 
lograr una reducción en los tiempos de lavado, que actualmente son 6 horas para un CIP normal, y una reducción de los tiempos por extensión del CIP (tiempo gastado adicional a las 6 horas, que generalmente se encuentra entre 3 y 5 horas) lo cual representa la segunda causa de tiempo perdido en la zona de evaporación, para de esta manera, aumentar el tiempo real invertido en la producción de leche en polvo.

Paralelo a esto, con la estandarización del CIP se alcanzan los siguientes beneficios; disminución de los costos de producción, aumento en la eficiencia de la evacuación de la leche, disminución de la carga microbiana por la reducción de los tiempos de almacenamiento, mejora de la calidad fisicoquímica y microbiológica de la leche cruda y la leche en polvo, disminución del consumo de energía, agua y soluciones de lavado, disminución de la generación de aguas residuales y residuos sólidos, aumento de la calidad higiénica del producto terminado, aumento del tiempo de las corridas de proceso, disminución de las paradas por aseo y consecución de condiciones de trabajo seguras para los operarios.

\section{MATERIALES Y MÉTODOS}

Concentración de las soluciones de lavado:

Se efectuó un procedimiento de preparación de las soluciones de lavado (soda caustica y ácido nítrico) aproximadamente 10 horas antes de iniciar el aseo a partir de soluciones concentradas al $50 \%$ mediante la adición de agua hasta lograr concentraciones del 2 al 3\% para el ácido y del 3 al $4 \%$ para la soda, las cuales fueron almacenadas en tanques independientes bajo recirculación para garantizar la homogenización de las mismas.

Se evaluó la evolución de la concentración de las soluciones de soda caustica y ácido nítrico tomando muestras de las mismas mientras recirculaban por los equipos, con el fin de determinar si su concentración se mantenía estable en los rangos inicialmente planteados (soda del 3 - 4\% y ácido del 2 - 3\%). Los análisis se realizaron mediante titulación con ácido clorhídrico $0.01 \mathrm{~N}$ e hidróxido de sodio $0.01 \mathrm{~N}$. Las muestras de ácido se tomaron cada 2 minutos mientras recirculaba por los equipos (durante 20 minutos), mientras que las muestras de soda se tomaron cada 5 minutos mientras recirculaba por los equipos (durante 3 horas).

Temperaturas de las soluciones de lavado: Las temperaturas de proceso del área de pulverización se consiguen inyectando vapor a los equipos por medio de 3 termocompresores los cuales tienen las posiciones de presión establecidas para lograr las temperaturas adecuadas durante el procesamiento de leche en polvo, sin embargo, durante el lavado CIP no se garantizaba la temperatura de las soluciones recirculadas ya que los termocompresores no se ubicaban en posiciones adecuadas ni estandarizadas. Se realizaron diferentes ensayos abriendo 0 cerrando los tres termocompresores con el fin de establecer en que posiciones se garantizaba la temperatura de recirculación de soda en un rango de 70 a $80^{\circ} \mathrm{C}$ censadas en las termocuplas de los 4 efectos del evaporador.

Caudales de proceso: Se realizaron diferentes ensayos ubicando el caudalimetro de los equipos en diferentes posiciones con el fin de determinar el caudal máximo posible a utilizar en el pasterizador para arrastrar las partículas adheridas y el caudal máximo en el evaporador que permitiera un buen lavado pero que impidiera la inundación del equipo pese al tamaño del mismo. Para lograr un caudal máximo en el pasterizador se realizo la instalación de una bomba en la línea de proceso que alcanzara los $60 \mathrm{l} / \mathrm{h}$.

Tiempo de recirculación de soda: Se realizaron diferentes lavados CIP con distintos tiempos de recirculación de soda caustica en el pasterizador, con el fin de determinar un tiempo mínimo que garantizara una limpieza eficiente y que no colocara en riesgo al equipo o al proceso productivo de leche en polvo.

La eficiencia de la limpieza se determinó teniendo en cuenta los siguientes parámetros: Duración de la corrida del proceso de producción de leche en polvo siguiente al CIP realizado, filtraciones realizadas en el equipo luego de terminar el CIP, verificación de ausencia de ATP en el equipo luego del lavado 
CIP mediante el uso del bioluminometro LIGHTNING y cantidad de impurezas recolectadas durante el CIP a la salida de los filtros del pasterizador.

Los diferentes tiempos de recirculación de soda en el evaporador y el pasterizador se establecieron de la siguiente manera:

- Ensayo 1: 1 hora de recirculación en el evaporador y 3 horas de recirculación en el pasterizador

- Ensayo 2: 1 hora de recirculación en el evaporador y 2.5 horas de recirculación en el pasterizador

- Ensayo 3: 1 hora de recirculación en el evaporador y 2 horas de recirculación en el pasterizador

Cada uno de estos ensayos se realizó garantizando condiciones iguales de proceso (Temperaturas de recirculación de las soluciones, caudales de proceso, tiempos de cada etapa y concentraciones de las soluciones).

Tiempo de corrida del proceso de elaboración de leche en polvo: Se cuantificó el tiempo de corrida del proceso de elaboración de leche en polvo luego de cada CIP realizado a diferentes tiempos de recirculación de soda en el pasterizador $(3,2.5$ y 2 horas), lo cual estuvo determinado por la llegada de la temperatura de la chaqueta del pasterizador a $120{ }^{\circ} \mathrm{C}$ (la temperatura de pasterización arranca en $92^{\circ} \mathrm{C}$, a medida que avanza la corrida de proceso la capa de costra leche que se va formando en el serpentín del pasterizador va aumentando y actúa como interferencia en la transferencia de calor, por tal motivo la temperatura de la chaqueta del pasterizador se va incrementando con el fin de vencer la interferencia de la capa de costra y garantizar la pasterización de la leche a $92^{\circ} \mathrm{C}$. Se decidió parar la corrida de proceso cuando se alcanzara una temperatura de $120^{\circ} \mathrm{C}$ en la chaqueta del pasterizador).

Impurezas descargadas por los filtros postpasterización: Durante cada proceso de CIP realizado, se recogió la totalidad de impurezas descargadas por los filtros postpasterización y se pesaron en una balanza analítica para estimar la cantidad de sucios generados durante la corrida que son removidos con el proceso de CIP realizado con los diferentes tiempos de recirculación de soda en el pasterizador (3, 2.5 y 2 horas).

Filtraciones: Una vez terminado el lavado CIP en los equipos se realizó una filtración de la última agua recirculada en el equipo utilizando filtros CHARM 0.5 micrometros, con el fin de evaluar la calidad del proceso mediante la retención de posibles partículas en un filtro de 0.5 micrómetros teniendo en cuenta los diferentes tiempos de recirculación de soda en el pasterizador (3, 2.5 y 2 horas).

Luminometría: Luego de cada proceso de lavado CIP se tomaron muestras con el bioluminómetro LIGHTNING con el fin de determinar la calidad higiénica mediante verificación de ausencia de ATP en los filtros postpasterización. Se tuvieron en cuenta los CIP realizados a diferentes tiempos de recirculación de soda en el pasterizador (3, 2.5 y 2 horas).

Microbiología de los equipos lavados con sistema CIP: Una vez finaliza el CIP se lleva a cabo un proceso de desinfección mediante la recirculación de agua a $98^{\circ} \mathrm{C}$ por los equipos. Con el fin de determinar si el equipo queda microbiológicamente limpio con el CIP actualmente efectuado se realizaron análisis de mesófilos, coliformes fecales y totales en las superficies de los equipos.

Los análisis de mesófilos se realizaron mediante frotis directo en una superficie de 10 x $10 \mathrm{~cm}$ utilizando escobillón, se incubó en caldo $\mathrm{BHI}$ a $32^{\circ} \mathrm{C}$ durante 6 horas, luego se sembró en petrifilm de manera directa y en dilución a la -2 y se incubó a $32^{\circ} \mathrm{C}$ durante 2 días.

Los análisis de coliformes fecales y totales se realizaron mediante frotis en una superficie de $10 \times 10$ con escobillón incubado a $32^{\circ} \mathrm{C}$ durante 6 horas en caldo BHI y luego sembrado en agar chromocult (medio selectivo para coliformes fecales y totales; las colonias de totales se visualizan de color rosado, mientras que las colonias de fecales se visualizan de color morado) e incubado a $37^{\circ} \mathrm{C}$ durante 2 días. 


\section{RESULTADOS Y DISCUSIÓN}

elpasterizadorysedeterminósu

Concentraciones de las soluciones de lavado: Se tomaron muestras de soda y ácido durante su recirculación en el evaporador y en

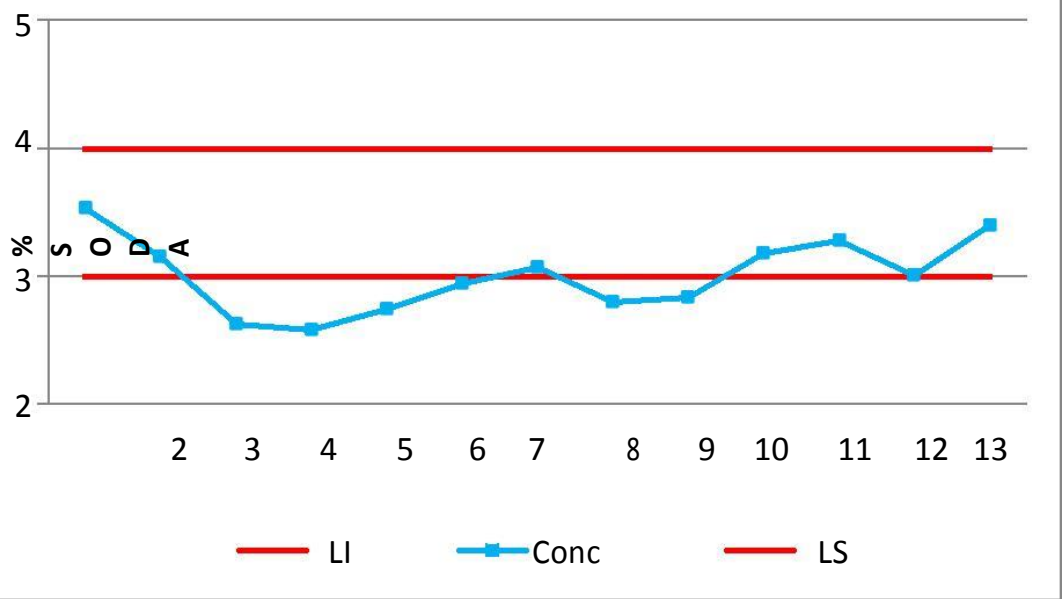

Figura 1. Evolución de la concentración de la soda durante la recirculación en el evaporador.

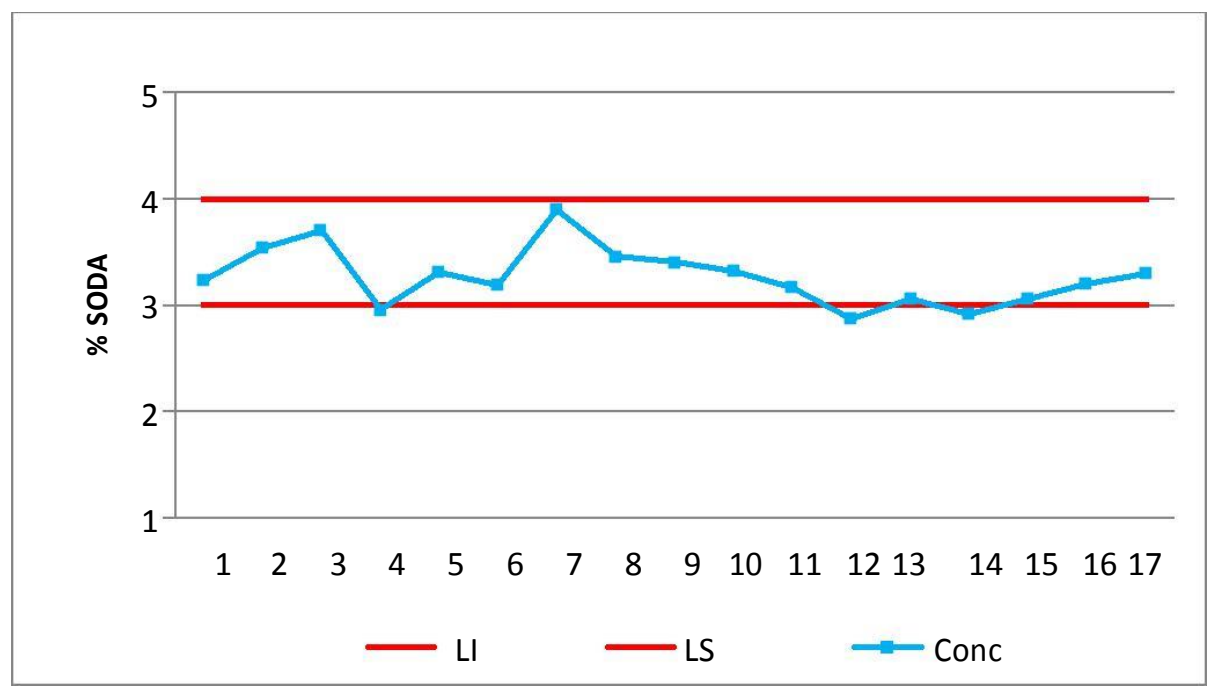

Figura 2. Evolución de la concentración de soda durante la recirculación en el pasterizador. 


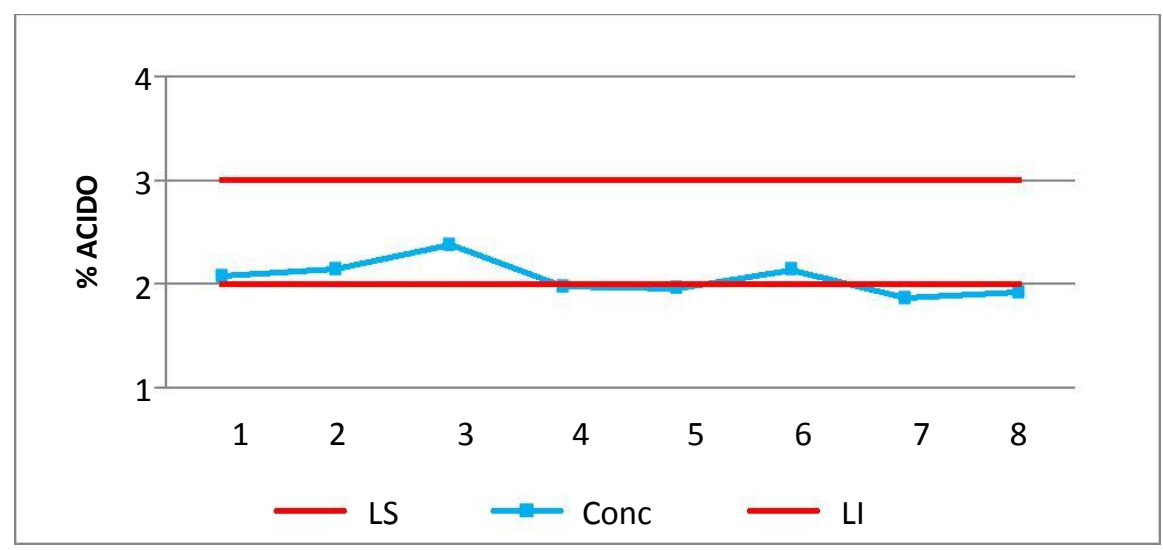

Figura 3. Evolución de la concentración de ácido durante la recirculación en el evaporador.

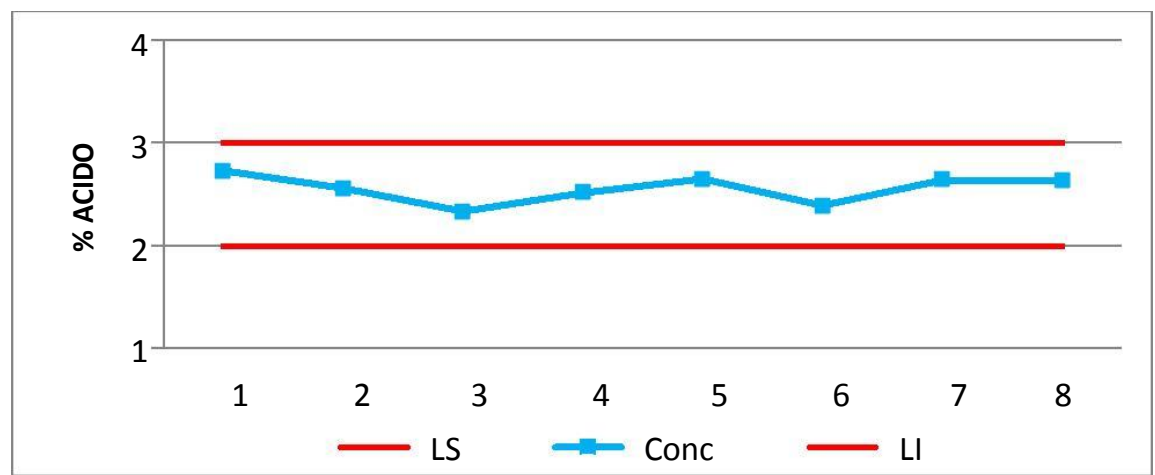

Figura 4. Evolución de la concentración del ácido durante la recirculación en el pasterizador.

Las concentraciones de las soluciones de lavado durante la recirculación en el pasterizador se mantienen estables en el rango de preparación (soda de $3-4 \%$ y ácido de $2-3 \%$ ), mientras que las soluciones en el evaporador recirculan a concentraciones ligeramente inferiores, esto puede deberse posiblemente a la cantidad de agua que queda en el equipo luego de los enjuagues debido al gran volumen de los mismos lo cual impide que el agua sea evacuada totalmente y diluye a la solución de lavado disminuyendo su concentración, sin embargo las concentraciones se mantuvieron notablemente más estables que con el procedimiento que existía anteriormente donde las soluciones recirculaban a concentraciones significativamente inferiores a los niveles deseados.

Temperaturas de las soluciones de lavado: Se realizaron diferentes ensayos variando las posiciones de los 3 termocompresores con el fin de encontrar las posiciones adecuadas que garantizaran las temperaturas requeridas. EI siguiente juego de posiciones de los termocompresores permitió alcanzar la temperatura adecuada durante la recirculación de la soda en los 4 efectos:

Termocompresor 1: Cerrado

Termocompresor 2: 0.4 bares

Termocompresor 3: Cerrado

En la figura 5 se puede observar la evolución de la temperatura de la soda durante la recirculación en los cuatro efectos del evaporador en un CIP realizado. 


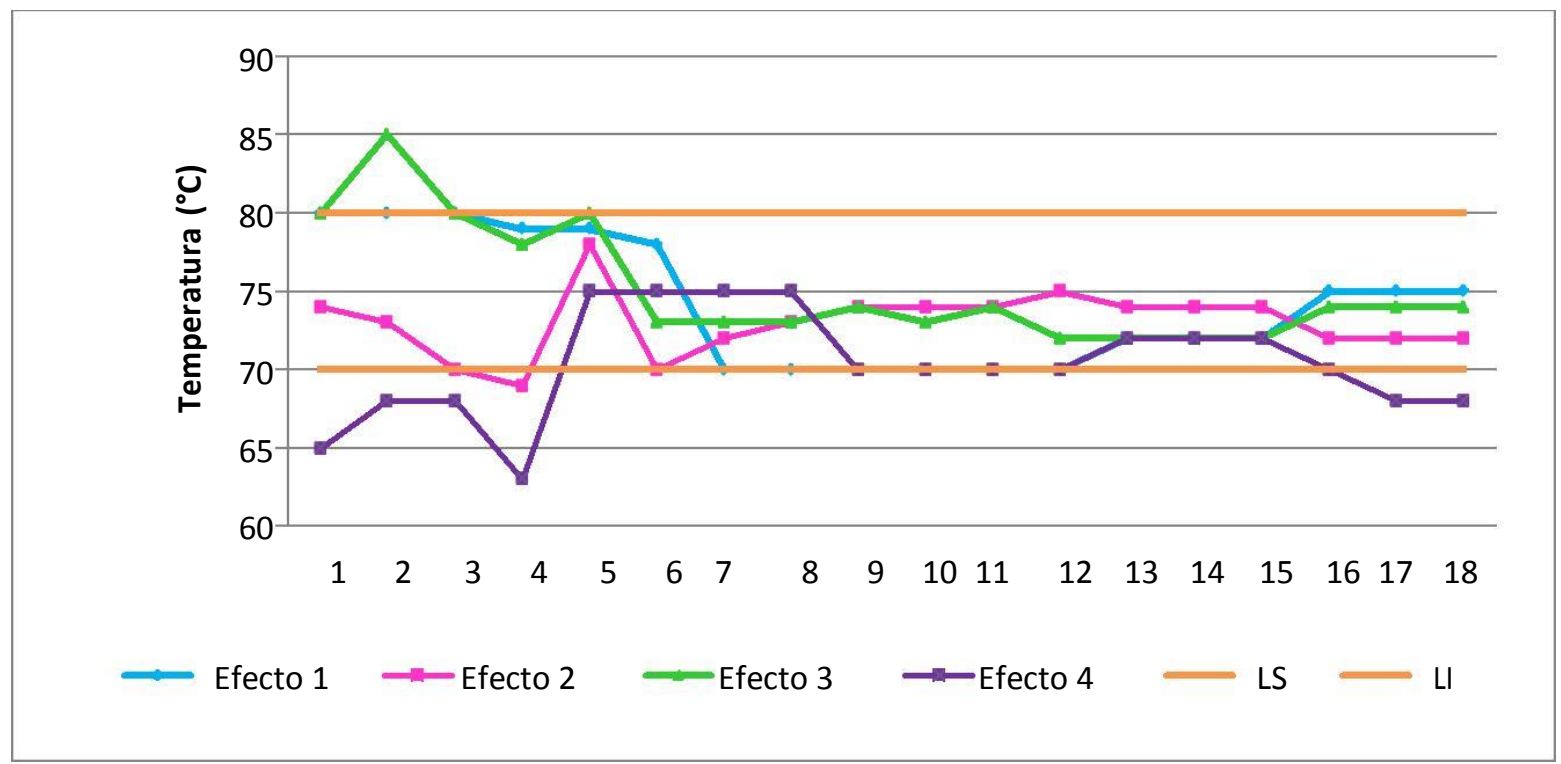

Figura 5. Temperatura de recirculación de soda en los 4 efectos del evaporador.

Se encontró una combinación adecuada de las posiciones de los termocompresores que permitió alcanzar las temperaturas establecidas en los 4 efectos del evaporador, en la figura 5 se puede observar que las solución de soda recircula durante todo el aseo a temperaturas entre 70 y $80^{\circ} \mathrm{C}$. El efecto 4 ó finalizador describe por lo general el comportamiento más bajo en temperatura ya que es el que está más alejado del vapor proveniente de los termocompresores. Al principio las temperaturas se muestran inestables pero a partir de los 10 minutos se alcanza la estabilidad. Con estas posiciones se alcanzan temperaturas durante la recirculación de soda entre 70 y $80^{\circ} \mathrm{C}$, las cuales están en conformidad con las especificaciones.

Caudal del proceso: El caudal de proceso en el evaporador se estableció mediante diferentes ensayos con flujos distintos, se determinó que cuando el flujo sobrepasaba los 15000 L/h se producía una inundación con las soluciones de lavado o de enjuague en los efectos del evaporador aumentando de esta manera el tiempo de evacuación de las soluciones, mientras que en el pasterizador se logró con la instalación de una nueva bomba un flujo máximo de $60000 \mathrm{l} / \mathrm{h}$ que facilita el arrastre de la costra de leche adherida al equipo.

Tiempo del proceso CIP: Se determinó el menor tiempo de recirculación de soda que garantizara una limpieza eficiente en el pasterizador teniendo en cuenta los siguientes factores:

Tiempo de corrida del proceso de leche en polvo luego del CIP realizado: El tiempo de corrida del proceso de elaboración de leche en polvo siguiente al CIP realizado da una idea de la calidad del lavado anterior, ya que si el lavado se hace siempre en igualdad de condiciones se espera que el tiempo de corrida siguiente sea similar al anterior, de lo contrario se puede tener un indicio de si el lavado anterior fue eficiente 0 no.

En la gráfica 6 se muestran los tiempos de corrida siguientes a los CIP realizados a diferentes tiempos de recirculación de soda en el pasterizador (3, 2.5 y 2 horas). 
Omar Pérez, Estandarización condiciones de lavado

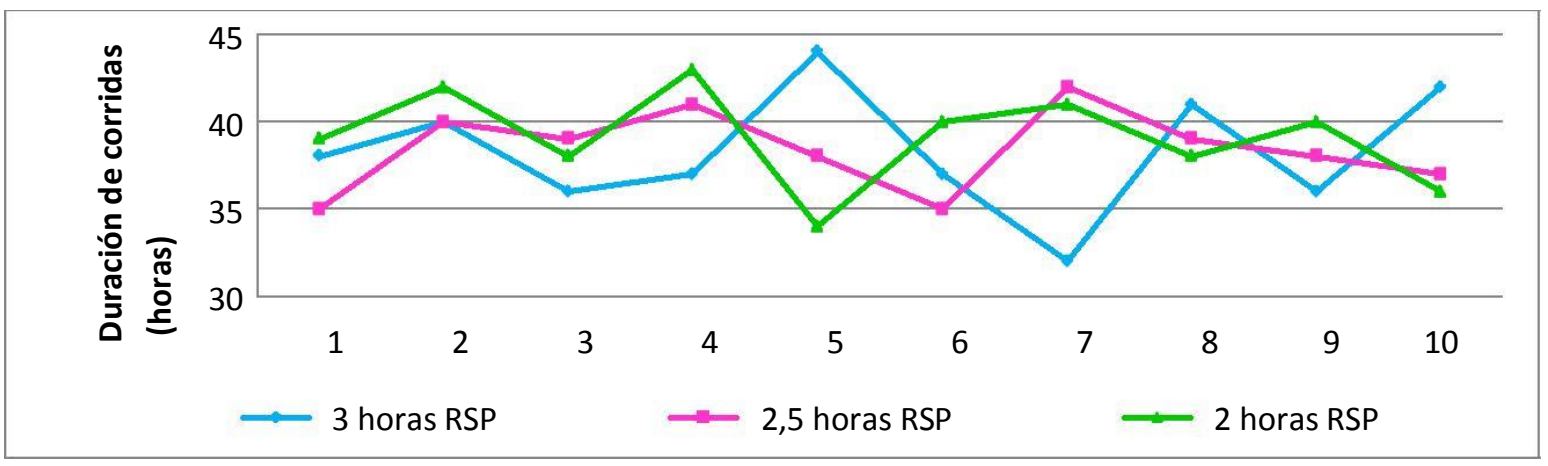

Figura 6. Tiempo de corrida de procesamiento de leche

en polvo luego de CIP realizados con diferentes tiempos de recirculación de soda en el pasterizador.

Se cuantificó el tiempo de duración de la corrida de proceso siguiente al CIP realizado y se determinó que en general las corridas se encontraban entre 30 y 40 horas sin importar el tiempo de duración de la recirculación de soda en el pasterizador, es decir no hubo influencia significativa de este factor en la duración de las corridas de proceso.

Tabla 1. Cantidad de impurezas recogidas a la salida de los filtros postpasterización durante varios CIP realizados con diferentes tiempos de recirculación de soda en el pasterizador.

\begin{tabular}{|c|c|c|c|c|c|}
\hline $\begin{array}{c}\text { Tiempo recirculación } \\
\text { de soda en el } \\
\text { pasterizador }\end{array}$ & \multicolumn{5}{|c|}{$\begin{array}{c}\text { Cantidad de impurezas recogidas en los } \\
\text { filtros pospasterización (gramos) }\end{array}$} \\
\hline 3 horas & 245 & 320 & 274 & 310 & 290 \\
\hline 2.5 horas & 246 & 324 & 403 & 200 & 256 \\
\hline 2 horas & 315 & 268 & 216 & 264 & 300 \\
\hline
\end{tabular}

En la tabla 1 se muestra la cantidad de impureza recolectadas en aseos realizados con los distintos tiempos de recirculación de la soda en el pasterizador, lo cual corresponde a la totalidad de restos de leche seca acumulada en el equipo durante la corrida de proceso de leche en polvo que es arrastrada durante el lavado CIP influenciado mayoritariamente por la acción de la soda caustica.

Filtraciones: se observaron diferentes filtraciones realizadas en los filtros postpasterización teniendo en cuenta los diferentes tiempos de recirculación de soda en el pasterizador (3, 2.5 y 2 horas).

Los resultados evidencian que no existe diferencia significativa entre las filtraciones realizadas bajo los tres tiempos de recirculación de soda en el pasterizador $(3,2.5$ y 2 horas) y que el equipo queda en similares condiciones de limpieza bajo los tres tratamientos.

Luminometría: Una limpieza efectiva debe remover residuos alimenticios y una correcta sanitización deberá asegurar la lísis y muerte bacteriana. La ausencia de ATP es, por lo tanto, un reflejo exacto de la limpieza de la superficie. Los luminómetros utilizan la bioluminiscencia de ATP para detectar residuos de alimentos y microorganismos que queden en el equipo procesador después de la limpieza e higienización. Por su alta sensibilidad, su reproductibilidad y el hecho de que está libre de sustancias interferentes, el luminómetro se convierte en la manera ideal 
de vigilar la eficacia de los procedimientos de limpieza en la industria de alimentos [3].

A continuación se muestran los resultados de la luminometría realizada en los filtros postpasterización luego de los aseos realizados con los diferentes tiempos de recirculación de soda en el pasterizador.

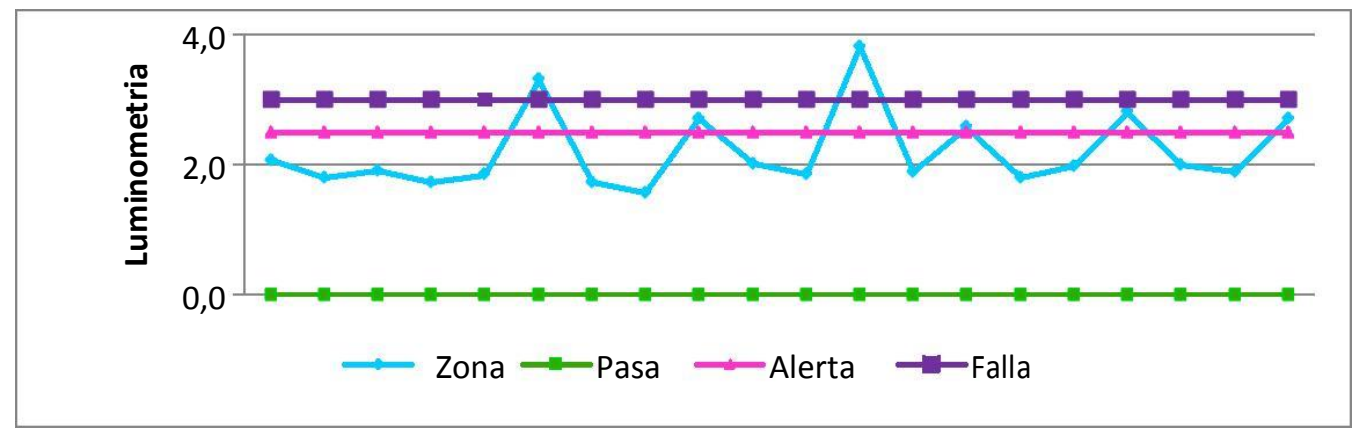

Figura 7. Luminometría realizada en los filtros postpasterización en un aseo de 3 horas de recirculación de soda en el pasterizador.

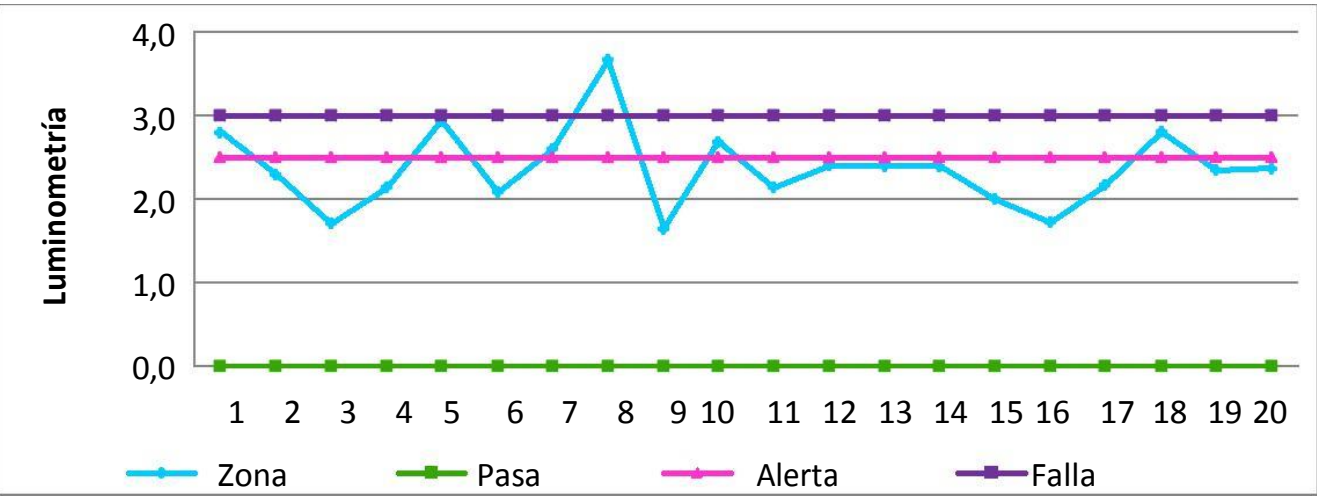

Figura 8. Luminometría realizada en los filtros postapasterización en un aseo de 2,5 horas de recirculación de soda en el pasterizador.

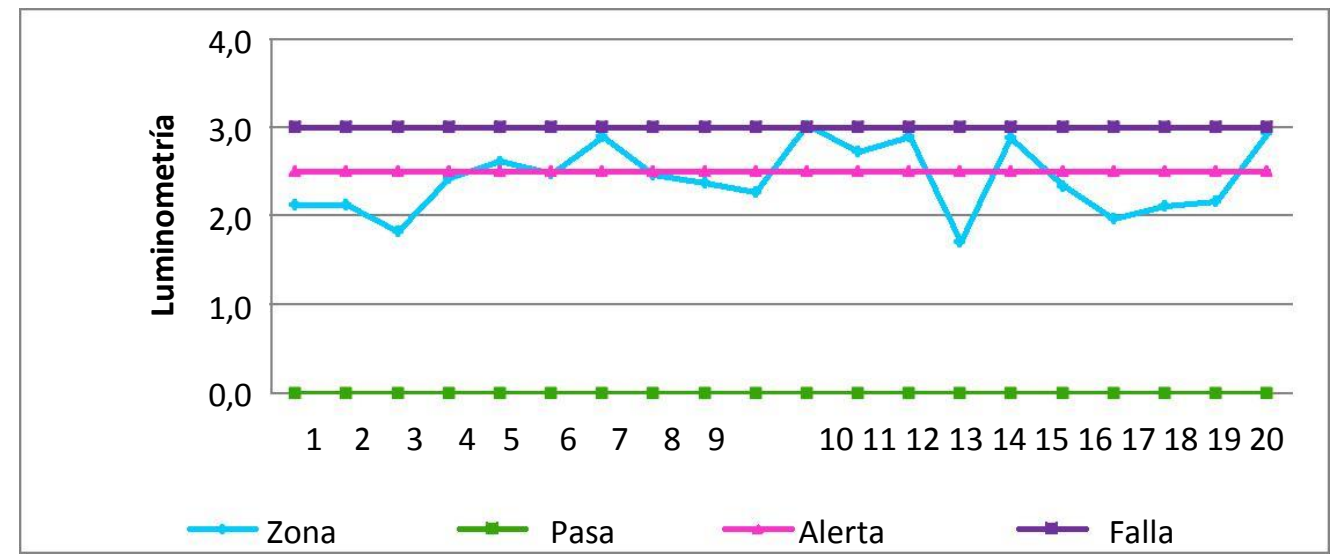

Figura 9. Luminometría realizada en los filtros postapasterización en un aseo de 2 horas de recirculación de soda en el pasterizador.

Los fotones o unidades fundamentales de emisión de luz son medidos por el luminómetro y convertidos en zonas. Las zonas de limpieza abarcan un margen desde 0 hasta 7.5 , las 
superficies limpiadas a fondo que usualmente se encuentran en las plantas procesadoras de alimentos, producen resultados inferiores a la zona 2.5 (pasa), los valores entre la zona 2.6 y 3 indican alerta (alerta) y por encima de 3 indican que el equipo o la zona analizada esta sucia (falla) [3].

En las anteriores figuras se puede observar que bajo las tres condiciones de CIP realizados (recirculación de soda en el pasterizador $3,2.5$ y 2 horas) se presentaron niveles de falla similares, y que aún con el menor tiempo de recirculación de soda (2 horas) los resultados de luminometría son aceptables, quedando el equipo higiénicamente limpio.

\section{Microbiología de los equipos lavados con sistema CIP.}

En la siguiente tabla se muestran los resultados de los análisis microbiológicos realizados a los equipos luego de finalizar el CIP.

FC: Filtros de concentrado FP:

Filtros Postapasterizaciòn.

Tabla 2. Mesófilos, coliformes totales y fecales en las superficies de los equipos lavados con CIP

\begin{tabular}{|c|c|c|c|}
\hline $\begin{array}{c}\text { Punto de } \\
\text { muestreo }\end{array}$ & $\begin{array}{c}\text { Mesófilos } \\
\text { (ufc/ml) }\end{array}$ & $\begin{array}{c}\text { Coliformes } \\
\text { fecales } \\
\text { (ufc/ml) }\end{array}$ & $\begin{array}{c}\text { Coliformes } \\
\text { totales } \\
\text { (ufc/ml) }\end{array}$ \\
\hline FC & 12 & $<1$ & $<1$ \\
\hline FC & 8 & $<1$ & $<1$ \\
\hline FC & $<1$ & $<1$ & $<1$ \\
\hline FC & $<1$ & $<1$ & $<1$ \\
\hline FC & 3 & $<1$ & $<1$ \\
\hline FP & 12 & $<1$ & $<1$ \\
\hline FP & $<1$ & $<1$ & $<1$ \\
\hline FP & $<1$ & $<1$ & $<1$ \\
\hline FP & $<1$ & $<1$ & $<1$ \\
\hline FP & $<1$ & $<1$ & $<1$ \\
\hline & & &
\end{tabular}

De la tabla 2 (anexo)se puede observar que hubo un recuento significativamente bajo de mesófilos en los equipos, posiblemente debido a una contaminación durante el muestreo o la siembra y hubo total ausencia de coliformes fecales o totales, lo cual confirma que luego de la desinfección el equipo si queda microbiológicamente limpio.

\section{CONCLUSIONES}

Se pudo determinar que no existe ningún riesgo para el equipo ni para el proceso de leche en polvo bajar el tiempo de recirculación de la soda en el pasterizador de 3 a 2 horas, ya que las variables analizadas mostraron comportamientos similares bajo los 3 tiempos de recirculación de soda en el pasterizador, lo cual demuestra que no es justificable desde ningún punto de vista un tiempo de recirculación y cabe la posibilidad de poder disminuir aún más este tiempo sin tener consecuencias negativas.

Se logró la reducción del tiempo total invertido en el proceso de CIP, pasando de 6 horas a 4,5 horas y de las extensiones por CIP hasta 2,56 horas, lo cual implica un ahorro de 24 horas al mes, que al ser invertido en la producción de leche en polvo y no en el CIP representa una ganancia adicional de \$62'371.584 mensuales.

Se logró la estabilidad de las condiciones en las que actualmente se realiza el CIP como concentraciones, temperaturas, caudales y tiempos de proceso.

Las condiciones actuales en las que se realiza el CIP garantizan la eficiencia del aseo, pese a esto hubo un incremento considerable del consumo de las soluciones de soda y ácido ya que se asegura mejor estabilidad de las mismas durante su recirculación, lo cual se vio contrarrestado por la disminución del consumo de antiespumante, el cual es el insumo más costoso (18 veces más que la soda) por tal motivo se logró una disminución de los costos por CIP relacionado con el consumo de insumos equivalente a $\$ 872.835,2$ mensuales. Los resultados microbiológicos evidencian que con el proceso de CIP realizado en la planta el equipo queda microbiológicamente limpio, lo cual garantiza que no se pone en riesgo el producto elaborado.

\section{REFERENCIAS}

[1]. Chmielewski y Frank, J. 2003. Biofilm Formation and Control in Food Processing Facilities. Institute of Food Technologists. 
Comprehensive Reviews in food Science and Food Safety. Vol. (2) pag: $22-32$.

[2]. Harper, J y Spillan, M. 2004. Cleaning and sanitizer Food plant equipment. [En línea] Disponible en Internet: http://class.fst.ohiostate.edu/FST401/Information/Cleaning\%2 Oand\%20Sanitizing.doc . [Accedido 18/11/2008].

[3]. Idarraga, E. 2004. Determinación de la calidad higiénica de los equipos lavados con sistema CIP en la planta Colanta San Pedro. Bacterióloga. Escuela de Bacteriología y laboratorio clínico, Colegio Mayor de Antioquía.

[4]. Monken, A y Winston, I. 2002. Milking System Cleaning and Sanitizing: Troubleshooting milk bacteria counts. West Agro. Kansas City Missouri. National Mastitis Council Regional Meeting Procedings.

[5]. Seiberling, D. 2004. Typical cleaning programs and procedures. [En línea]. Disponible de Internet: http://www.seiberling4cip.com/prg\&proc.htm [Accedido 14/09/2008].

[6]. Tetra Pack Processing Systems AB. 2003. Manual de industrias lácteas. Ed. Acríbia S.A. Primera Edición. Madrid España, pag: $403-413$.

[7]. Tybor, P y Gilson, W. 2004. Cleaning and sanitizing in the milking system. Gerogia, Estados Unidos. Disponible en Internet: $<$ http: //Plaptnsup01/PASCION/Biblioteca/Limpie za\%20ydesinfeccion. 